\title{
Management of Primary Insomnia Using Cognitive Behavior Therapy
}

\author{
Vikas Sharma, Gopal Ch. Mahakud, SPK Jena \\ Department of Applied Psychology, University of Delhi, Delhi, India
}

DOI No:

Indian J Sleep Med 2013; 8.3, 123-128

\begin{abstract}
Background:Sleep difficulty is a major issue in recent times. The problem is more pronounced in metropolitan areas attributing to more stressful environment. Research indicates that there are different approaches to the management of insomnia and Cognitive behavior therapy (CBT) is one of the preferred treatment modalities.

Objective:To determine the symptoms related to primary insomnia and impact of cognitive behavior therapy.

Method:In the present study a group of 15 participants (Age range 20-30) who fulfilled the criteria of primary insomnia were administered 10 sessions based on cognitive behavior therapy. All the participants were voluntary and informed consent for the study was obtained. Frequency of sessions was once a week. Four components of CBT:Stimulus control instructions, sleep hygiene education, relaxation and cognitive therapy were implemented.

Results:Improvements in insomnia related variables such as sleep onset latency, number of nightly awakenings, total sleep time, wake up after sleep onset, sleep quality and sleep efficiency were measured before and after the intervention. The results indicate improvements in above mentioned insomnia related variables.
\end{abstract}

Key words: Insomnia, Cognitive behavior therapy, Sleep diary, Relaxation, Sleep quality

S leep difficulty or insomnia is a major challenge which adversely affects quality of life. Insomnia is not only restricted to elderly population but also widespread among the adults. Approximately $30 \%$ of adult from different countries have been suffering from different symptoms of insomnia such as difficulty initiating sleep, difficulty maintaining sleep, waking up too early, and poor quality of sleep ${ }^{1}$. Insomnia can be classified as primary or secondary ${ }^{2}$. Insomnia is not an

\section{Correspondence Ȧddress}

Dr Vikas Sharma

Department of Psychology, Room No-208, Department of Applied Psychology, University of Delhi, South Campus, New Delhi-110021, India email: vikasdelhi83@hotmail.com independent disorder but may be associated with various other psycho-physiological problems. Studies ${ }^{3-6}$ indicate that stress is an important factor contributing to insomnia. Earlier the role of stressors is also highlighted by $3 \mathrm{P}$ model ${ }^{7-8}$, where the authors used the concepts of predisposing, precipitating and perpetuating factors to describe the pathogenesis of insomnia. They stated that predisposing factors such as age, gender, hyperarousability, family/personal history of insomnia and medical and psychiatric illnesses are the risk factors that make the individual more vulnerable to insomnia. Similarly precipitating factors like separations, hospitalization or death of a loved one or daily hassles are those events that trigger or coincide with the onset of insomnia. Finally, the authors emphasized several perpetuating factors (i.e. behavioral practices that 
maintain sleep difficulty, mental worrying about sleeplessness or misconception about sleep in sustaining sleep difficulties over time.

The cognitive behavioral therapy for insomnia is a multi-component approach. That consists of stimulus control therapy, sleep restriction, cognitive therapy, relaxation based interventions, and sleep hygiene education. Stimulus control instructions are behavioural treatment aimed to address the problem of insomnia. This technique is useful in managing conditioned insomnia as proposed by various researchers ${ }^{9-10}$. The chronic insomniacs have a tendency to become aroused at the bed time contributing to sleep onset difficulties. The process of going to bed develops into automatic trigger for frustration and arousal. The main objective of the stimulus control therapy is to train the insomniac to re-associate the bed and bedroom with rapid sleep onset by curtailing sleep incompatible activities ${ }^{10-11}$. This process involves going to bed only at sleep time, getting out of bed when unable to sleep in 20-25 minutes and returning to bed only when sleepy, postponement of bed time until sleep is imminent, maintaining a regular wake up time and using bed only for the purpose of sleep and not for watching television.

Sleep restriction therapy (SRT) addresses the disruption of homeostatic and circadian processes which is commonly found among patients suffering from chronic insomnia. In the absence of adequate sleep, poor sleepers often increase their time in bed a strategy which more often leads to fragmented and poor quality of sleep. Sleep restriction therapy consists of setting up a sleep window which is aimed at reducing the time spent in bed to the actual amount time asleep ${ }^{12-13}$. For example, if an individual reports sleeping for an average of $5 \mathrm{hrs}$ and spent 8 hours in bed, the initial prescribed "sleep window" would ne 5 hours. This is subsequently adjusted on the basis of sleep efficiency (ratio of total sleep time to total time in bed). Faulty beliefs and attitudes about sleep have been associated with insomnia and challenging it may break a vicious cycle of anxiety and arousal ${ }^{14-15}$. Further they proposed that cognitive therapy seeks to alter faulty beliefs, expectations, and attributions about sleep and insomnia. The basic premise of this approach is that appraisal of a given situation can trigger negative emotions such as fear and anxiety that are incompatible with sleep. To short circuit this cycle cognitive therapy is designed to identify and correct misconceptions about sleep.
Greater physiological arousal among patients with insomnia led to the application of relaxation and biofeedback techniques to the disorder ${ }^{16}$. Further a study ${ }^{17}$ affirmed that experience of cognitive or physiological arousals during the pre-sleep period contribute to longer sleep-onsetlatency and shorter total sleep time.Sleep may be facilitated by activities that promote mental calm and physical relaxation. There are several subtypes of relaxation based interventions, some methods, such as progressive muscle relaxation, which reduces muscle tension by sequentially tensing and relaxing the main muscle groups, autogenic-training, which promotes somatic relaxation by inducing sensations of warmth and heaviness ${ }^{18}$. In this regard biofeedback focuses primarily on reducing somatic arousal ${ }^{19}$. Biofeedback is designed to train a patient to control some physiological parameters through visual or auditory feedback. Sleep hygiene refers to the habits and practices of everyday life that affect sleep $\mathrm{p}^{20-21}$. Sleep hygiene education targets health practices (e.g. diet, exercise, substance use) and environmental factors (e.g. light, noise, temperature, and mattress) that may be either detrimental or beneficial to sleep. Although these factors are rarely severe enough to be the primary cause of chronic insomnia, they may complicate an existing sleep problem and hinder treatment progress.

Although a number of studies have been conducted on insomnia by using various approaches, few studies focused on the management of insomnia using cognitive behavior therapy. The inadequate facility in Indian health care sector renders very negligible research on insomnia patients specifically following cognitive behavior therapy. Therefore the study was planned to find out the effects of cognitive behavior therapy in primary insomnia.

\section{Method}

Objective: To determine the effectiveness of cognitive behavior therapy in reducing the severity of insomnia in a group of individuals suffering from primary insomnia.

Design: Before-After intervention without control group design was followed for the present study. During the pre-assessment, the researchers studied sleep related variables such as sleep onset latency, number of nightly awakenings, total wake time, total sleep time, percentage of sleep efficiency, and sleep quality which are mostly related to primary insomnia. In the present study, all the assessments were conducted by using Sleep Diary ${ }^{22}$. The 
sleep diary measures were taken for a period of two weeks. After assessing sleep related variables all the participants were interviewed by the researchers to identify their individual concerns related to insomnia. During the intervention, the researchers applied the components of cognitive behavior therapy which included stimulus control instructions, sleep hygiene education, relaxation training and cognitive therapy. Sleep restriction therapy was not used in the present study because it is associated with day time sleepiness and researchers intended to make the intervention more acceptable to the participants without interfering with their regular activities. During the post assessment the sleep related variables were assessed again. Statistical analysis of the study followed descriptive analysis by using mean scores and standard deviation for pre and post assessment to determine the effectiveness of Cognitive behavior therapy.

Sample: Fifteen voluntary participants suffering from primary insomnia were recruited in the study who fulfilled the combined criteria of International Classification of Sleep Disorders ${ }^{23}$ and of the Diagnostic and Statistical Manual of Mental Disorders, $4^{\text {th }}$ edition ${ }^{24}$. For the present study informed consent from all participants were obtained. They were assured that the information collected for the study will be kept confidential and will be used only for research purpose. The criteria included Inability to sleep within 30 minutes of going to bed or awakening during the night for 30 minutes at least 3 nights per week, difficulty with sleep causing marked distress or significant deterioration in day time functioning (e.g. fatigue, disturbed mood, performance deficits) and problem endurance for a minimum of 6 months. Clients having insomnia with severe major depression, another serious psychiatric disorder, presence of sleep disorder other than insomnia (e.g. sleep apnea, periodic limb movements), presence of another illness affecting the immune system, regular use of psychotropic medication were excluded from the study. No participant in the study reported using any medications for insomnia.

Material: Sleep diary ${ }^{22}$ was the major tool used in this study. The diary consists of a set of forms to estimate the sleep parameters such as sleep onset latency, frequency of awakenings during the night, time spent asleep and some of the qualitative aspects of sleep.

Procedure: In this study brief clinical interview for diagnosing insomnia, which included use of diagnostic criteria for insomnia specified in DSM IV and International classification of Sleep disorders were used. The researchers used sleep diary for a period of two weeks to measure the variables related to their sleep difficulties. The clients were called to department of applied psychology laboratory, University of Delhi, Delhi, India for both assessment and intervention individually. The total duration of the intervention was approximately for a period of $2^{1 / 2}$ months. The entire intervention schedule was divided into 10 weekly sessions, each lasting for a period of about one hour. During the first and second sessions all the clients were given stimulus control instructions "use the bed and bedroom only for the purpose of sleep; go to bed only when you feel sleepy; lie in bed awake for no longer than 20 minutes else get up and sit on a chair; return to bed only when you feel sleepy and set the alarm for the same time each morning. Sleep hygiene education was then administered for a period of two sessions which included discontinuation of caffeine and nicotine especially before 4 to 6 hrs of bed time, avoid alcohol and drugs, avoid exercises before bedtime, minimize noise and high illumination in the bed room and keep the room temperature ideal, no pondering over the activities happened during the day nor planning about the coming day. To counter the physiological arousal of the clients, the researchers imparted applied relaxation training ${ }^{25}$ for a period of three weeks. The relaxation training was supervised by the researchers and participants were encouraged to practice it once every day in their home settings. The aim of relaxation training was to reduce physiological arousal which is a hindrance in sleep. Finally cognitive therapy was administered for a period of three weeks to deal with the irrational thinking and assumptions related to sleep. During cognitive therapy sessions, each participant's general beliefs related to their sleep problems were discussed and counterproductive beliefs were identified. Common dysfunctional thoughts were addressed during the intervention such as misconceptions concerning the causes of insomnia, misattributions or amplifications of its consequences, unrealistic sleep expectations, diminished perception of sleep and mistaken beliefs about the predictability of sleep. After this, cognitive restructuring was undertaken. The post intervention assessment similar to pre-intervention assessment was then carried out.

Results: Cognitive behavior intervention improved the sleep related outcome measures. The results of the present study indicated that after the implementation of cognitive 
behaviour therapy for a period of 10 sessions only, lead to improvements among the patients of insomnia. The variable related to insomnia i.e. sleep onset latency during the pre-intervention assessment was $37.26 \pm 3.00$ minutes which was reduced to $31.30 \pm 3.48$ minutes after the successful completion of interventions. The number of nightly awakenings and wake up after sleep onset are related to the sleep maintenance problems. The result of the study indicates that of mean number of nightly awakenings was $1.06 \pm 0.58$ during the pre intervention sessions which was reduced to $0.40 \pm 0.28$ during post intervention sessions. The study revealed that the average wake up after sleep onset time was $(95 \pm 18.39)$ minutes before the cognitive behaviour intervention which declined to $(63.00 \pm 15.14)$ minutes at post intervention. The result shows that the mean total wake time which is obtained by adding sleep onset latency time and wake up after sleep onset time of the participants during pre intervention was $132.26 \pm 18.7$ minutes that came down to $94.90 \pm 13.95$ during post intervention assessment.

From the present study it was found that participants mean total sleep time was $4.90 \pm 0.58$ hours before intervention which was increased to $5.40 \pm 0.54$ hours after cognitive behavior therapy. The mean total bed time was a $7.13 \pm 0.36$ hour which was reduced to $6.94 \pm 0.47$ hours. The result indicates an increase in total sleep time and a reduction of total bed time leading to an increase in percentage of sleep efficiency from $69.5 \%$ to $78.5 \%$. In the present study mean sleep quality was $1.4 \pm 0.68$ before the intervention which was increased to $2.5 \pm 1.16$ after the intervention. The details of the pre-intervention and post intervention effects are depicted in Table- 1 .

\section{Discussion and Conclusions}

The mean difference of sleep onset latency (5.96) from the pre-intervention assessment score $(37.26 \pm 3.00)$ minutes to $(31.30 \pm 3.48)$ minutes indicates the improvement of sleep onset latency of participants due to the effects of the intervention. A similar study ${ }^{26}$ on 32 participants suffering insomnia using CBT also indicated that sleep onset latency improved with the help of cognitive behavior therapy.Further the results of the study revealed that mean number of nightly awakenings was $(1.06 \pm 0.58)$ during the pre intervention sessions which was reduced to $(0.40 \pm 0.28)$ during post intervention sessions. The result demonstrates improvements in this variable related to insomnia. In the study ${ }^{27}$ on 26 people suffering insomnia using CBT found that the mean numbers of nightly awakenings reduced 2 times during pre intervention to 0.40 times after the intervention. This study partially supported the present work of researchers. It can be concluded from numbers of researches based on CBT thatit reduces the nightly awakenings of patients suffering from insomnia.

The study indicated that the average wake up after sleep onset time was $(95 \pm 18.39)$ minutes before the cognitive behaviour intervention which declined to $(63.00 \pm 15.14)$ minutes at post intervention. The mean difference of (32.00 minutes) indicates an improvement to the present insomnia related variable. Effectiveness of CBT on primary insomnia has been critically illustrated in a systematic review of seven studies published from 1993 to 2004 and stated that CBT is anusefull modality for the management of primary insomnia ${ }^{28}$. The authors found that CBT was effective

Table 1: Pre and post Intervention mean scores of clients on primary and secondary sleep outcome measures

\begin{tabular}{|l|l|l|l|l|l|l|}
\hline S. No. & Sleep parameters & \multicolumn{2}{|c|}{ Pre intervention } & \multicolumn{2}{c|}{ Post intervention } & \multicolumn{2}{l|}{ Mean Differences } \\
\hline & & & Mean & SD & Mean & \multicolumn{2}{l|}{ SD } \\
\hline 1 & Sleep onset latency (Min) & 37.26 & 3.00 & 31.30 & 3.48 & 5.96 \\
\hline 2 & No. of Nightly awakenings & 1.06 & 0.58 & 0.40 & 0.28 & 0.66 \\
\hline 3 & Wake up after Sleep onset (mins) & 95.00 & 18.39 & 63.00 & 15.14 & 32.00 \\
\hline 4 & Total wake time (mins) & 132.26 & 18.73 & 94.90 & 13.95 & 37.36 \\
\hline 5 & Total sleep time (hrs) & 4.90 & 0.58 & 5.40 & 0.54 & 0.50 \\
\hline 6 & Time in bed (hrs) & 7.13 & 0.36 & 6.94 & 0.47 & 0.19 \\
\hline 7 & Sleep efficiency (\%) & 69.50 & 5.40 & 78.50 & 5.05 & 9.00 \\
\hline 8 & Sleep quality & 1.40 & 0.68 & 2.50 & 1.16 & 1.10 \\
\hline
\end{tabular}

Indian Journal of Sleep Medicine (IJSM), Vol. 8, No. 3, 2013 
in reducing wake up after sleep onset. This indicates that the participants spent less time wake during the night after intervention ${ }^{26,28}$.

The result of the present study revealed that participants mean total sleep timebefore intervention was $(4.90 \pm 0.58$ hours $)$ which was increased to $(5.40 \pm 0.54)$ hours after cognitive behavior therapy.The total sleep time is used as an important criterion to diagnose insomnia. Generally the insomniacs tend to get less than 6 hours of sleep. The increase of sleep time might be due to the effect of intervention. A similar study ${ }^{29}$ indicates the improvement of total sleep time after cognitive behavior therapy. Sleep efficiency is calculated by dividing total sleep time by total bed time and multiplying it with 100 . Thus the sleep efficiency can enhance by raising the total sleep time or reducing the total bed time. The mean total bed time was a $7.13 \pm 0.36$ hour which was reduced to $6.94 \pm 0.47$ hours. The result indicates an increase in total sleep time and a reduction of total bed time leading to an increase in percentage of sleep efficiency from $69.5 \%$ to $78.5 \%$. In the present study mean sleep quality was $1.4 \pm 0.68$ before the intervention which was increased to $2.5 \pm 1.16$ after the intervention. in this context the study ${ }^{30}$ highlighted the significant improvement of sleep efficiency from $78.8 \%$ to $85.1 \%$ with the effect of cognitive behavior therapy indicating an improvement of $6.3 \%$ whereas the present study reveal an improvement of $9 \%$ (mean difference) in sleep efficiency among participants. The sleep quality is related to the subjective aspect of sleep. In the present study mean sleep quality was $1.4 \pm 0.68$ before the intervention which was increased to $2.5 \pm 1.16$ after the intervention. It indicatesthat the subjective experiences about the sleep improved after the cognitive behavior therapy. The findings of the present study can be confirmed with a recent similar study ${ }^{31}$ related to sleep quality aspect.

Conclusion: The objective of the present study was to determine the effectiveness of the cognitive behavior therapy in reducing the severity of symptoms related to insomnia in individuals suffering from primary insomnia. Cognitive behavior therapy led to improvements of those measures which are indicators of good sleep. The present study therefore establishes that cognitive behavior therapy can be a preferred intervention modality for the management of insomnia.

Limitations:The major limitations of the present study can be explained in terms of its small sample size $(\mathrm{n}=15)$.
Researchers suggest replication of the study on larger sample cases with the control group. The statistical analysis for present study followed a descriptive analysis only by recording the mean scores and the standard deviation scores. The study could be made more reliable by using parametric inferential statistics like ANOVA and student's ' $t$ ' test. Further, this study utilized different components of cognitive behavior therapy so the individual effect of each component cannot be determined. More studies using single components may be conducted.

\section{References}

1. Ancoli-Israel S, Roth T.Characteristics of insomnia in the United States: results of the 1991 National Sleep Foundation Survey. I. Sleep 1999; 22, (2): 347-53.

2. Erman MK. Introduction to Chronic Insomnia: Classification and Prevalence. Primary Psychiatry 2007; 14, (5):27-31.

3. Kales A, Kales J.Evaluation and treatment of insomnia. Oxford University Press; New York: 1984.

4. Kales A, Soldatos CR, Kales JD. Review Sleep disorders: insomnia, sleepwalking, night terrors, nightmares, and enuresis. Annals of Internal Medicine 1987; 106, (4): 582 92.

5. Ohayon MM. ZulleyJ.Correlates of global sleep dissatisfaction in the German population. Sleep 2001; 24, (7): 780-7.

6. Jernelöv S, Lekander M, Kaldo V. Efficacy of a behavioral self-help treatment with or without therapist guidance for co-morbid and primary insomnia -a randomized controlled trial, BMC Psychiatry 2012;12, (5): 1-23.

7. Spielman AJ. Assessment of insomnia.Clinical Psychology Review.1986; 6:11-26.

8. Spielman AJ, GlovinskyPB.The varied nature of insomnia. In: Hauri PJ, editor. Case studies in insomnia. Plenum Press; New York: 1991:1-15.

9. Bootzin RR. Stimulus control treatment for insomnia.Proceedings of American Psychological Association 1972; 7: 395-6.

10. Schutte-Rodin S, Broch L, Buysse D, Dorsey C,Sateia M. (2008).Clinical guideline for the evaluation and management of chronic insomnia in adults. Journal Clinical Sleep Medicine 2008; 4, (5):487-504.

11. Bootzin RR, Epstein D, Wood JM. Stimulus control instructions, In: P. Hauri (Ed.) Case studies in insomnia, New York, N Y: Plenum Press, 1991:19-28

12. Spielman AJ, SaskinP,Thorpy MJ. Treatment of chronic insomnia by restriction of time in bed.Sleep 1987; 10: 4556.

13. Glovinsky PB, Spielman AJ. How did I get insomnia? In: Glovinsky PB, Spielman AJ. The Insomnia Answer: A Personalized Program for Identifying and Overcoming the

Indian Journal of Sleep Medicine (IJSM), Vol. 8, No. 3, 2013 
Three Types of Insomnia. New York, NY: Berkley Publishing Group; 2006:51-82.

14. Morin CM. Insomnia: Psychological assessment and management, New York, The Guilford Press; 1983.

15. Harvey AG, Tang NKY, Browning L. Cognitive approaches to insomnia. Clinical Psychology Review 2005; 25: 593611.

16. Monroe L. Psychological and physiological difference between good and poor sleepers.Journal of Abnormal Psychology 1967; 72: 255-264.

17. Tang NKY Harvey AG. Effects of cognitive arousal and physiological arousal on sleep perception. Sleep 2004; 27, (1):69-78

18. Nicassio P, Bootzin RA. A comparison of progressive relaxation and autogenic training as treatment for insomnia.Journal of Abnormal Psychology, 1974; 83: 253 60.

19. HauriPJ.Treating psychophysiological insomnia with biofeedback.Archives of General Psychiatry 1981; 752758.

20. Hauri PJ. Case studies in insomnia. New York: Plenum medical book 1991

21. Stepanski EJ,WyattJK. Use of sleep hygiene in the treatment of insomnia. Sleep Medicine Reviews, 2003; 7, (3): 215 225.

22. Coates TJ, Killen JD, George G, Manchini E, Silverman S, Thoresen C. Estimating sleep parameters: A multitraitmultimethod analysis. Journal of Consulting and Clinical Psychology1982:50, (3), 345-352.

23. American Sleep Disorder Association, International Classification of Sleep Disorders (ICSD); Diagnostic and Coding Manual. Rochester, MN: Author, 1997.
24. American Psychiatric Association, Diagnostic and statistical Manual of Mental Disorders-IVth Edition-Washington: DC. Author, 1994.

25. Ost LG. Applied Relaxation: description of a coping technique and review of controlled studies. Behaviour Research and Therapy 1987; 25: 397-410.

26. Verbeek IH, Konings GM, Aldenkamp AP, Declerck AC, Klip EC. Cognitive behavioral treatment in clinically referred chronic insomniacs: group versus individual treatment. Behaviour Sleep Medicine, 2006;4(3):135-51.

27. $\mathbf{G}^{3} \mathbf{u} \mathbf{z} \mathbf{k} \mathbf{0}-\mathbf{W}$ êgieln i k M, Jakuszkowiak WojtenK, Wiglusz MS, Cuba ${ }^{3}$ a WJ, Landowski J. The efficacy of Cognitive-Behavioural Therapy (CBT) as related to sleep quality and hyperarousal level in the treatment of primary insomnia. Psychiatria Danubina 2012; 1: 51-55.

28. Wang MY, Wang SY, Tsai PS.Cognitivebehavioural therapy for primary insomnia: a systematic review. Journal of Advanced Nursing 2005; 50 (5): 553-564.

29. Morin CM, Culbert JP, SchwartzSM.Nonpharmacological interventions for insomnia: a meta-analysis of treatment efficacy. American Journal Psychiatry1994; 151 (8):117280.

30. Edinger JD, Wohlgemuth, WK, Radtke, RA, Marsh GR, QuillianRE.Cognitive behavioral therapy for treatment of chronic primary insomnia: a randomized controlled trial. JAMA 2001; 2858 (14): 1856-164.

31. Van Houdenhove L, Buyse B, Gabriels L, Van Diest I, Van den Bergh O. (2010). Cognitive-behavioural therapy for primary insomnia: effectiveness in a clinical setting. Tijdschr Psychiatry2010; 52 (2): 79-88. 